\title{
Thermal transformation of polyacrylonitrile deposited on SBA-15 type silica
}

\author{
Effect on adsorption capacity of methyl-ethyl ketone vapor
}

\author{
Rafał Janus • Piotr Natkański • Anna Wach • \\ Marek Drozdek $\cdot$ Zofia Piwowarska $\cdot$ \\ Pegie Cool · Piotr Kuśtrowski
}

CEE-TAC1 Conference Special Issue

(C) The Author(s) 2012. This article is published with open access at Springerlink.com

\begin{abstract}
Thermogravimetry, diffuse reflectance infrared Fourier transform spectroscopy, and X-ray photoelectron spectroscopy (XPS) were used for the studying of thermally induced structural changes of polyacrylonitrile (PAN) deposited on the surface of SBA-15 type mesoporous silica. Polymer was introduced onto the support by the precipitation polymerization of acrylonitrile in aqueous suspension of SBA-15. Low temperature transformation (to $723 \mathrm{~K}$ ) of the deposited PAN was analyzed. It was found that at about $523 \mathrm{~K}$, exothermic cyclization of polymer chains to the so-called ladder form of PAN occurred. However, the total cyclization of PAN required higher carbonization temperatures, at which gradual dehydrogenation followed by graphitization was initiated. XPS revealed that the cyclic form of PAN and a relatively large amount of carbonyl species, formed during the carbonization of the PAN/SBA-15 composite at $623 \mathrm{~K}$, were responsible for the high sorption capacity in the methylethyl ketone (MEK) vapor elimination. The efficiency in the MEK adsorption was also influenced by the content of PAN-derived carbon deposited on the SBA-15 surface.
\end{abstract}

Keywords Thermal transformation of polyacrylonitrile SBA-15 support · Thermogravimetry $\cdot$ X-ray photoelectron spectroscopy · Diffuse reflectance Fourier transform spectroscopy · Adsorption of volatile organic compounds

R. Janus · P. Natkański · A. Wach · M. Drozdek ·

Z. Piwowarska · P. Kuśtrowski ( $\square)$

Faculty of Chemistry, Jagiellonian University, Ingardena 3, 30-060 Kraków, Poland

e-mail: kustrows@chemia.uj.edu.pl

P. Cool

Department of Chemistry, University of Antwerpen,

Universiteitsplein 1, 2610 Wilrijk, Belgium

\section{Introduction}

Polyacrylonitrile (PAN) homopolymer and acrylonitrile (AN)-containing copolymers are widely used in textile industry for the production of synthetic fibers with properties similar to wool and silk. As a high-melting, rigid and spinnable duroplast, PAN is a very useful raw material for the preparation of microporous activated carbon and carbon fibers by controlled pyrolysis followed by an appropriate chemical and/or thermal activation [1-4]. PAN-derived activated carbons are applied as the adsorbents for the removal of heavy metal ions and organic pollutants from aqueous solutions as well as volatile organic compounds (VOCs) from exhaust gases [5-7]. The mechanism of the PAN thermal transformation during carbonization in an inert atmosphere includes three stages: (1) cyclization of PAN chains and the formation of aromatic structures (543-623 K); (2) dehydrogenation and condensation of cyclic structures (673-773 K); (3) loss of nitrogen and formation of graphitelike domains (873-1573 K) [8-10]. Typically, the PAN carbonization is carried out at temperatures providing graphitization. The resulting carbonizate is subsequently treated with oxidizing agents to form surface functional groups responsible for the adsorption properties.

In the present study we have demonstrated highly effective adsorbents of VOCs obtained by thermal decomposition of PAN deposited on SBA-15 type mesoporous silica developing the available surface of carbonaceous species. This study deals with the decomposition mechanism of PAN stabilized by the $\mathrm{SiO}_{2}$ support. Since high temperature transformations of PAN are well described, we consider the low temperature range (473-723 K) of transition stages. This temperature range has been found to be appropriate for obtaining promising adsorbents of methyl-ethyl ketone (MEK) vapor chosen as a probe 
molecule representing polar VOCs [10]. For the investigation of thermal transformation of PAN layers highly dispersed on the mesoporous silica support, thermogravimetric (TG) analysis, diffuse reflectance infrared Fourier transform spectroscopy (DRIFTS) as well as X-ray photoelectron spectroscopy (XPS) have been applied.

\section{Experimental}

\section{Materials}

Mesoporous silica SBA-15 was synthesized, using a molar gel composition: 1.00 tetraethyl orthosilicate (TEOS): 0.02 Pluronic 123: $5.58 \mathrm{HCl}$ : $182.55 \mathrm{H}_{2} \mathrm{O}$, according to the procedure described earlier [11]. $4.0 \mathrm{~g}$ of poly(ethylene oxide)-block-poly(propylene oxide)-block-poly(ethylene oxide) triblock copolymer $\left(\mathrm{EO}_{20} \mathrm{PO}_{70} \mathrm{EO}_{20}\right.$, Pluronic P123) was dissolved in $150 \mathrm{~cm}^{3}$ of $1.6 \mathrm{M} \mathrm{HCl}$ solution, and then $9.14 \mathrm{~cm}^{3}$ of TEOS was slowly dropped. The obtained suspension was stirred at $318 \mathrm{~K}$ for $8 \mathrm{~h}$ and subsequently aged at $353 \mathrm{~K}$ for $15 \mathrm{~h}$. The solid product was filtered, washed with distilled water, and dried at room temperature. In order to remove the organic template, the dried solid was calcined at $823 \mathrm{~K}$ for $8 \mathrm{~h}$ at a heating rate of $1 \mathrm{~K} / \mathrm{min}$.

PANx/SBA-15 composite materials with intended PAN/ silica mass ratios $(\mathrm{x})$ of $0.1,0.2,0.6,1.2$, and 2.0 were synthesized by radical polymerization of $\mathrm{AN}$ in aqueous slurry of SBA-15 [12]. Typically, $3 \mathrm{~g}$ of the preliminary dried support was introduced into a three-necked flask $\left(250 \mathrm{~cm}^{3}\right)$ equipped with a reflux condenser. Then, adequate volumes of water, freshly distilled AN, and an initiator (1.2\% solution of $2,2^{\prime}$-azobis(isobutyronitrile) hydrochloride (AIBA) in water) were added to achieve the total mixture volume of $100 \mathrm{~cm}^{3}$. The AIBA/AN molar ratio was kept at a constant level of 0.004 . The intended PAN/SBA-15 mass ratios were achieved using an appropriate $A N$ volume. The mixture was continuously purged with argon at a flow rate of $40 \mathrm{~cm}^{3} / \mathrm{min}$. The polymerization process was performed at $333 \mathrm{~K}$. Then, the obtained composite was isolated by filtration after $3 \mathrm{~h}$ of heating and stirring, washed with distilled water, and dried at $333 \mathrm{~K}$ overnight. Subsequently, the samples were carbonized in a tubular furnace at 523,573 , 623,673 , or $723 \mathrm{~K}$ for $4 \mathrm{~h}$ with a heating rate of $5 \mathrm{~K} / \mathrm{min}$. The inert gas (argon; grade 4.6) was passed by the oven at a flow rate of $40 \mathrm{~cm}^{3} / \mathrm{min}$ during the whole thermal treatment process and cooling down to room temperature.

\section{Characterization}

The powder X-ray diffraction (XRD) measurement was carried out using a Bruker D2 Phaser equipped with a LYNXEYE detector in the $2 \theta$ angle range of $0.8^{\circ}-5.0^{\circ}$.
The textural parameters of the parent SBA-15 molecular sieve were determined using low temperature adsorption of nitrogen at $77 \mathrm{~K}$. The sample was preliminarily outgassed at $473 \mathrm{~K}$ for $12 \mathrm{~h}$. The adsorption-desorption isotherm was collected in an ASAP 2010 (Micromeritics) instrument.

The obtained PAN/SBA-15 precursors were analyzed by the TG-SDTA method. The measurements were performed in a Mettler Toledo TGA/SDTA 851 instrument. Argon $\left(80 \mathrm{~cm}^{3} / \mathrm{min}\right)$ was passed by a sample $(\sim 10 \mathrm{mg})$ placed in a corundum crucible heated from 303 to $1273 \mathrm{~K}$ with a heating rate of $10 \mathrm{~K} / \mathrm{min}$.

The carbonized PAN/SBA-15 samples were examined by DRIFTS and XPS. For the DRIFTS measurements, samples were diluted to $2 \%$ by weight in potassium bromide and softly milled. The spectra were recorded on a Nicolet 6700 FTIR spectrometer. 200 scans were collected at a resolution of $4 \mathrm{~cm}^{-1}$.

The XPS measurements were performed with a Prevac photoelectron spectrometer equipped with a hemispherical analyzer VG SCIENTA R3000. The photoelectron spectra were taken using a monochromatized aluminum source $\mathrm{AlK} \alpha(E=1486.6 \mathrm{eV})$ and a low-energy electron flood gun (FS40A-PS) to compensate charge on the surface of nonconductive samples. The base pressure in the analysis chamber was $5 \times 10^{-9}$ mbar. The surface composition was studied on the basis of the areas and binding energies of $\mathrm{C}$ $1 s, \mathrm{~N} 1 s, \mathrm{O} 1 s$ and Si $2 p$ core levels. The binding energy values of measured regions were referenced to the $\mathrm{C}$ $1 s$ core level $(285.0 \mathrm{eV})$.

\section{Adsorption study}

About $0.1 \mathrm{~g}$ of a carbonized sample was weighed in a small glass vessel. The open vessel was kept in a dryer at $423 \mathrm{~K}$ for $30 \mathrm{~min}$ (a preliminary outgassing step). After drying, the mass of the sample was determined strictly, and the adsorbent was quantitatively transferred into a stainless steel adsorber with a total volume of $1.2 \mathrm{~cm}^{3}$. Subsequently, the adsorber was placed in an oven kept at $313 \mathrm{~K}$. The constant flow of carrier gas $\left(\mathrm{N}_{2}, 20 \mathrm{~cm}^{3} / \mathrm{min}\right)$, saturated with MEK vapor at $283 \mathrm{~K}$, was passed by the adsorber. When no signal changes were detected in a flame ionization detector (FID) connected to the adsorber outlet, dosing MEK was interrupted, and the system was purged by pure nitrogen for $30 \mathrm{~min}$ to remove the loosely adsorbed forms of ketone. Then, the adsorber was heated from 323 to $623 \mathrm{~K}$ at a heating rate of $15 \mathrm{~K} / \mathrm{min}$ in a flow of $\mathrm{N}_{2}$ $\left(20 \mathrm{~cm}^{3} / \mathrm{min}\right)$. The FID response was calibrated quantitatively by injections of various amounts of liquid MEK. This procedure enabled us to apply the intensity of the FID signal recorded during MEK desorption for the calculation of the real sorption capacity of the PAN/SBA-15 composites. 


\section{Results and discussion}

\section{SBA-15 characterization}

The structural and textural properties of the synthesized silica support were determined by XRD and $\mathrm{N}_{2}$ adsorption methods. The collected isotherm is plotted in Fig. 1, whereas the pore size distribution and the low angle XRD pattern of SBA15 are shown in inset $a$ and $b$, respectively. The XRD pattern reveals a highly ordered, two-dimensional hexagonal structure (p6mm) with (100), (110), and (200) reflections at $1.03^{\circ}$, $1.73^{\circ}$, and $1.99^{\circ}$, respectively. The calculated hexagonal cell parameter $a$ is equal to $9.9 \mathrm{~nm}$. The shape of the isotherm recorded for the SBA-15 support can be classified as IV type according to the IUPAC classification pointing out that synthesized silica is a mesoporous material [13]. Furthermore, the pore size distribution calculated by the Barrett-JoynerHalenda (BJH) model following the adsorption branch of the isotherm confirms the mesoporous character of SBA-15. The majority of channels has diameters close to $6.1 \mathrm{~nm}$; however, these mesopores are additionally interconnected through micropores $\left(V_{\text {micro }}=0.118 \mathrm{~cm}^{3} / \mathrm{g}\right)$. The specific surface area of SBA-15 is equal to $722 \mathrm{~m}^{2} / \mathrm{g}$, while the total pore volume determined by the single-point method (at $p / p_{0}=0.98$ ) reaches $0.734 \mathrm{~cm}^{3} / \mathrm{g}$.

\section{Thermal transformation of PAN deposited on SBA-15}

\section{Thermal analysis}

A series of PAN/SBA-15 composites were synthesized by the deposition of various amounts of PAN on the SBA- 15 surface using the precipitation polymerization. In order to elaborate the mechanism of thermal decomposition of deposited



Fig. 1 Adsorption-desorption isotherm $\left(\mathrm{N}_{2}, 77 \mathrm{~K}\right)$ with pore size distribution (inset $a$ ) and low-angle XRD pattern (inset $b$ ) collected for the SBA-15 support

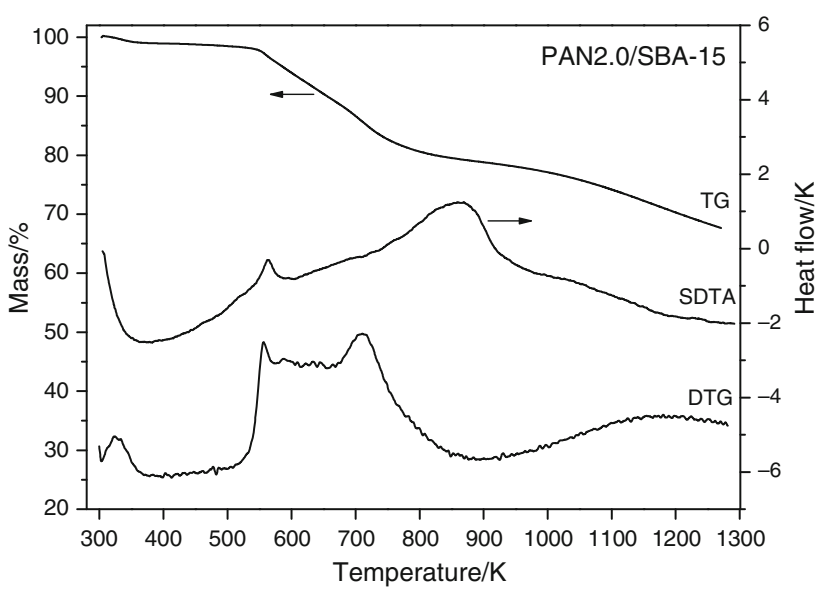

Fig. 2 TG, DTG, and SDTA curves recorded for the PAN2.0/SBA15 sample in the presence of argon

polymer, the sample containing the highest PAN loading (PAN2.0/SBA-15) was studied by TG. Moreover, DRIFTS and XPS measurements for PAN2.0/SBA-15 carbonized at different temperatures (523-723 K) were performed.

TG, DTG, and SDTA curves recorded for the PAN2.0/ SBA-15 composite in flowing argon are presented in Fig. 2. The decomposition of the sample in an inert atmosphere occurs in a few steps. The first one, observed at about $380 \mathrm{~K}$, is ascribed to the evacuation of physically bound water. The effect of mass loss in this temperature range is relatively low and does not exceed $2 \mathrm{wt} \%$. Raising temperature to about $523 \mathrm{~K}$ results in the exothermic effect of cyclization of polymer chain structure to the so called ladder form of PAN [14, 15]:

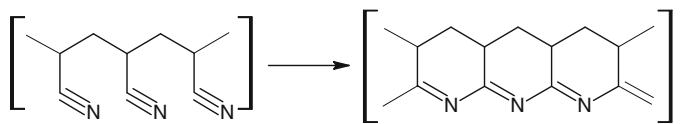

The slightly endothermic process is observed at temperatures above $600 \mathrm{~K}$. This effect is attributed to the dehydrogenation of "ladder" PAN structure leading to the formation of unsaturated condensed species [16]:

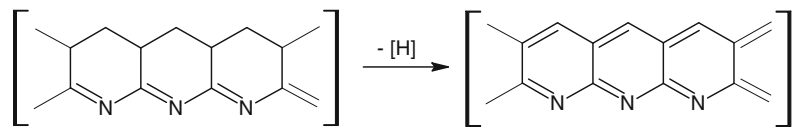

Finally, the last transformation takes place at temperatures higher than $900 \mathrm{~K}$. This step is ascribed to the deep condensation of PAN-derived domains connected with the loss of nitrogen and the appearance of graphitic-like structure:

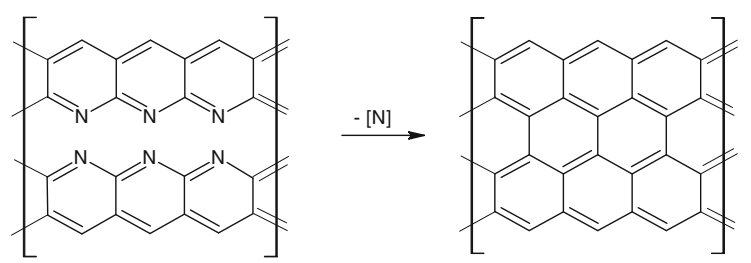




\section{FTIR spectroscopy}

To look into the structural transformations, which undergo at lower temperatures, the PAN2.0/SBA-15 composite was carbonized in the range of 523-723 K. The DRIFT spectra collected for PAN2.0/SBA-15 before and after the thermal treatment are comparatively shown with the spectrum of unmodified SBA-15 in Fig. 3.

The spectrum of the neat support shows the bands typical of silica. The sharp band at $3,740 \mathrm{~cm}^{-1}$ is attributed to the vibrations of isolated silanols, while the broad band at $3,000-3,700 \mathrm{~cm}^{-1}$ proves the presence of hydrogen-bonded silanol groups on the SBA-15 surface. The strong absorption bands observed at $810 \mathrm{~cm}^{-1}$ and in the region of $1,000-1,250 \mathrm{~cm}^{-1}$ correspond to the $\mathrm{Si}-\mathrm{O}$ stretching vibrations and dehydroxylated surface siloxane bridges $\left(1,240 \mathrm{~cm}^{-1}\right)$ [17]. The band at $960 \mathrm{~cm}^{-1}$ is assigned to the $\mathrm{Si}-\mathrm{OH}$ stretching vibrations. Moreover, the presence of surface water is detected by the band at $1,635 \mathrm{~cm}^{-1}$ corresponding to the bending vibration of adsorbed $\mathrm{H}_{2} \mathrm{O}$ [12].

In the spectrum collected for the PAN2.0/SBA-15 composite, beside the absorption bands attributed to the vibrations of silica, additional bands characteristic of the deposited polymer appear. The bands at 2,939 and $2,873 \mathrm{~cm}^{-1}$ are ascribed to the stretching vibrations of $\mathrm{CH}$ and $\mathrm{CH}_{2}$ groups in the polymer chains, respectively. Furthermore, the bands at 1,453 and $1,358 \mathrm{~cm}^{-1}$ are assigned to $\pi\left(\mathrm{CH}_{2}\right)$ and $\delta(\mathrm{CH})$ vibrations, respectively. The sharp band at $2,243 \mathrm{~cm}^{-1}$ is attributed to the stretching vibrations of $\mathrm{C} \equiv \mathrm{N}$ functional groups. It should be noticed that the intensity of band attributed to the vibrations of free silanol groups (at $3,740 \mathrm{~cm}^{-1}$ ) in the composite sample is significantly lower compared to that in unmodified SBA-15. This effect is caused by a strong interaction between the silica surface and polymer chains.

The treatment at raised temperatures causes a distinct decrease in the intensity of the nitrile vibration band (at $2,243 \mathrm{~cm}^{-1}$ ). This effect could have been brought about by the formation of the ladder form of PAN, and therefore, the bands appearing at $1,383,1,583$, and $1,613 \mathrm{~cm}^{-1}$, ascribed to the vibrations of conjugated $\mathrm{C}=\mathrm{C}$ and $\mathrm{C}=\mathrm{N}$ systems, are observed [12, 18]. Nevertheless, the intensities of these bands gradually decrease with increasing the carbonization temperature. Thus, we can conclude that the ladder form of PAN is stable in the temperature range of about 573-623 K. The decrease in the intensities of IR active vibrations of $\mathrm{CH}$ and $\mathrm{CH}_{2}$ with an increase in the treatment temperature proves the dehydrogenation of cyclic form of polymer.

In order to quantitatively analyze the observed changes, the measured reflectance $(\mathrm{R})$ has been recalculated into the Kubelka-Munk (KM) units using the following equation:

$K M=\frac{(1-R)^{2}}{2 R}$.

The intensities of $1,383,1,613$ and $2,243 \mathrm{~cm}^{-1}$ bands, expressed in KM units, are graphically compared on the right side of Fig. 3. The diagram shows that the sample carbonized at $573 \mathrm{~K}$ contains the greatest amount of the ladder form of PAN.

\section{$X$-ray photoelectron spectroscopy}

The conclusions based on TG analysis and DRIFT spectra have been supported by XPS measurements. The changes in the $\mathrm{C} 1 s$ and $\mathrm{N} 1 s$ core level spectra with increasing
Fig. 3 DRIFT spectra of the silica support and the PAN2.0/ SBA-15 composite and changes in the intensities of characteristic IR bands with increasing carbonization temperature

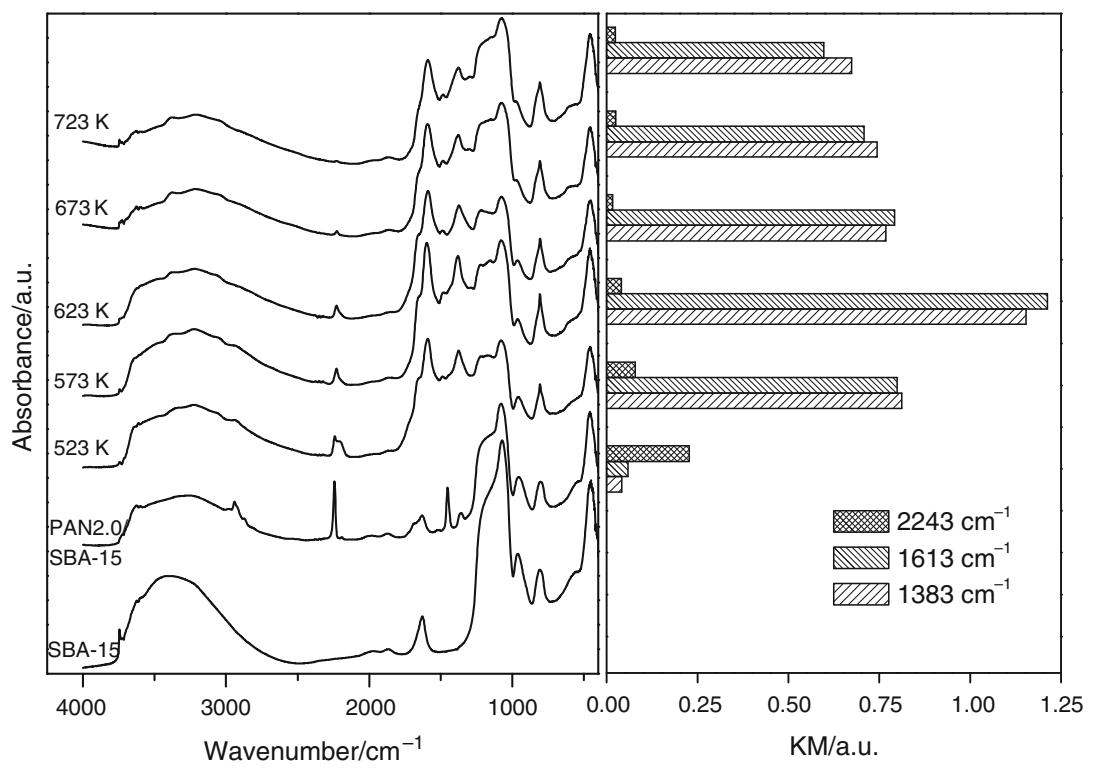


treatment temperature are shown in Fig. 4. The atomic concentration and binding energy values for $\mathrm{N}, \mathrm{C}, \mathrm{O}$, and $\mathrm{Si}$ species present on the surface of the PAN2.0/SBA-15 precursor and corresponding carbonizates are summarized in Table 1.

The $\mathrm{N} 1 s$ core level spectrum obtained for the non-carbonized sample shows a singular peak at $399.2 \mathrm{eV}$ indicating that nitrogen exists only in the cyano groups [19]. The spectra obtained for the carbonized samples were fitted with two different peaks. The signal at $398.6-398.7 \mathrm{eV}$ points out the presence of conjugated imines and a pyridinic-type structure [20] formed by the cyclization process of PAN chains, whereas the peak at $399.8-400.4 \mathrm{eV}$ comes from pyrrolic or pyridon- $\mathrm{N}$ moieties [21] formed during the dehydrogenation of cyclized PAN structure. For the sample
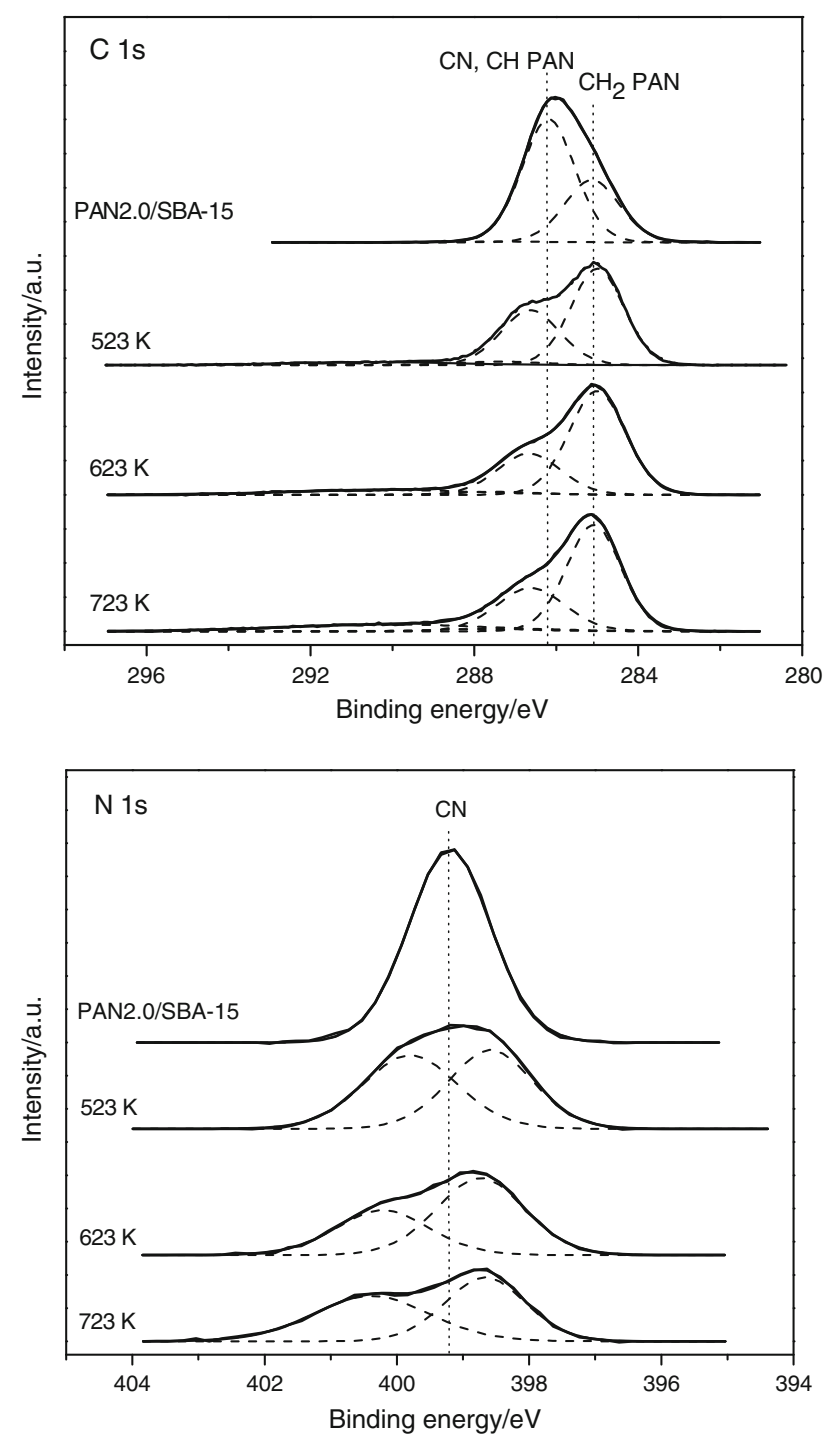

Fig. $4 \mathrm{C} 1 s$ and $\mathrm{N} 1 s$ regions of XPS spectra for the PAN2.0/SBA-15 sample before and after carbonization at 523-723 K carbonized at $523 \mathrm{~K}$ this latter peak is probably overlapped by the signal originating from non-cyclized PAN chains, which are still present on the composite surface. The intensities of measured $\mathrm{N} 1 s$ core level spectra decrease with the increasing carbonization temperature. The total amount of nitrogen is slightly lowered during the high-temperature treatment indicating slow graphitization.

The $\mathrm{C} 1 s$ spectrum for the non-carbonized composite contains three components with chemical shifts corresponding to: $\mathrm{CH}_{2}$ groups in PAN chain $(285.1 \mathrm{eV}) ; \mathrm{CH}$ and $\mathrm{C}=\mathrm{N}$ groups $(286.2 \mathrm{eV})$; and $\mathrm{C}=\mathrm{O}$ groups $(287.4 \mathrm{eV})$. Small amounts of carbonyl groups are probably formed during the radical polymerization of $\mathrm{AN}$ in an aqueous medium. For the carbonized PAN/SBA-15 composites, the optimum fitting is achieved by resolving each $\mathrm{C} 1 s$ region into four peaks [22]. The main peak with the binding energy of $285.0( \pm 0.1) \mathrm{eV}$ is attributed to aliphatic $(\mathrm{C}-\mathrm{C}$, $\mathrm{C}-\mathrm{H})$ and graphitic $\left(\mathrm{C}=\mathrm{C} \mathrm{s} p^{2}\right)$ carbons. The signal at 286.6 $( \pm 0.1) \mathrm{eV}$ corresponds to the carbon type present in the $\mathrm{C}=\mathrm{N}$ groups. The peak with a higher binding energy of $287.6( \pm 0.1) \mathrm{eV}$ is typical of carbon in carbonyl groups. The additional signal at about $290.1 \mathrm{eV}$, observed in the sample carbonized at $723 \mathrm{~K}$, is attributed to shake-up satellite due to $\pi-\pi^{*}$ transitions in aromatic rings [23]. It is noteworthy that the changes in the carbon species contribution are well correlated with the $\mathrm{N}$ content. Moreover, a significant increase in the content of $\mathrm{C}=\mathrm{O}$ groups after carbonization is observed. The maximum concentration of carbonyl species is found for the sample treated at $523 \mathrm{~K}$. The presence of these groups is additionally confirmed by the $\mathrm{O} 1 s$ spectra, in which the signal at $530.9( \pm 0.1) \mathrm{eV}$ attributed to oxygen in $\mathrm{C}=\mathrm{O}$ functionalities is detected.

\section{Sorption capacities of C/SBA-15 composites}

The carbonized PAN/SBA-15 composites were tested as adsorbents in the removal of MEK vapor. The influence of carbonization temperature on the sorption efficiency determined for the PAN0.1/SBA-15 sample is shown as inset in Fig. 5. It is noticed that the thermal treatment at $573 \mathrm{~K}$ results in obtaining the material that exhibits the best sorption capacity. Taking the TG, DRIFTS and XPS results into account, it should be assumed that the superior adsorption properties can be attributed to the presence of cyclized PAN structure deposited on the silica surface. Moreover, the promoting effect of carbonyl group cannot be excluded.

Besides the carbonization temperature, the content of PAN-derived carbon is also an important factor influencing the adsorption capacity of the PAN/SBA-15 composite. Figure 5 displays the effect of carbon loading on the adsorption capacities. As seen, high dispersion of carbon materials on the silica surface enhances the adsorption 


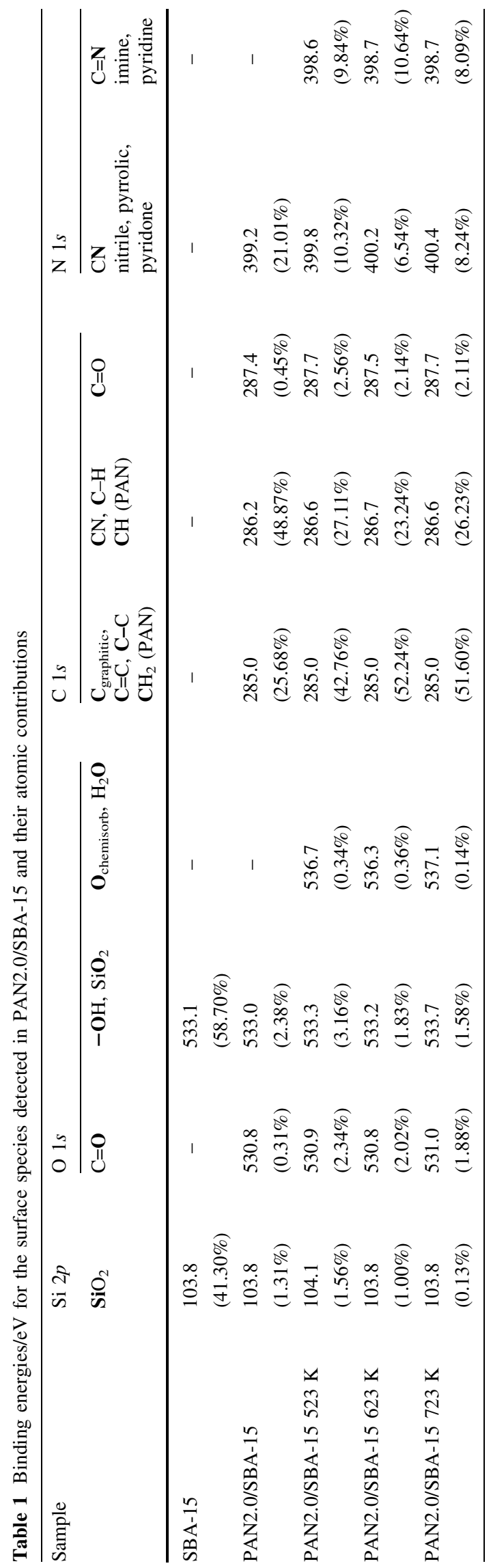

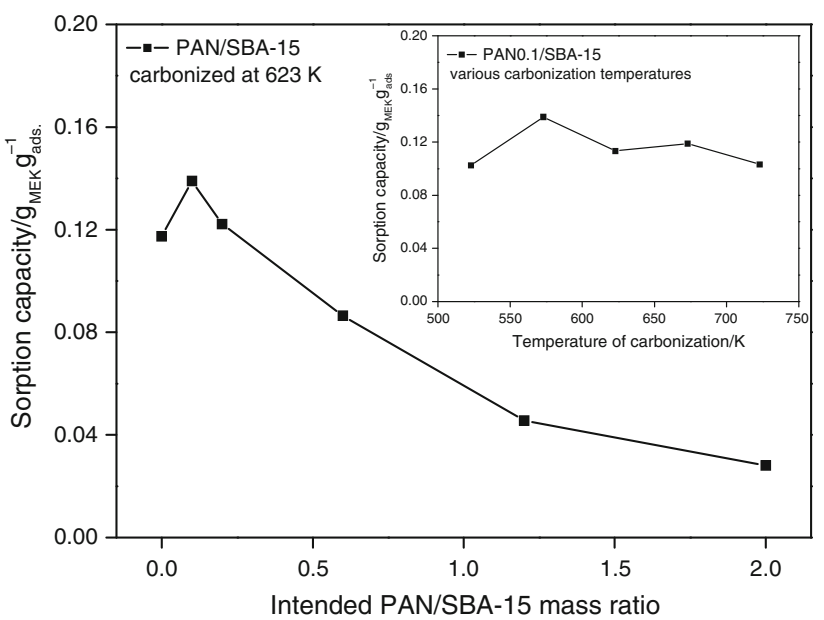

Fig. 5 Effect of carbonization temperature and PAN-derived carbon content on sorption capacity of methyl-ethyl ketone

effectiveness. It should be noted that carbon deposition on the SBA-15 support develops its sorption capacity. However, a higher amount of introduced PAN blocks the pore system, and the opposite effect is observed.

\section{Conclusions}

The low-temperature thermal transformation of PAN deposited on the SBA-15 mesoporous silica surface by the precipitation polymerization was investigated by $\mathrm{TG}$, DRIFTS, and XPS measurements. It was found that in the temperature range of 523-723 K cyclization of PAN followed by dehydrogenation of the cyclic form occurred. Carbonyl species were also formed. The increase in the carbonization temperature led to the deep dehydrogenation resulting in graphitization of the thermally treated material. These processes were not favorable for the high sorption capacity of the PAN-derived carbon supported on silica in the elimination of ketone vapor.

Acknowledgements This study was supported by the Polish Ministry of Science and Higher Education under the grant no. N N507 553238. Rafał Janus wishes to thank the Foundation for Polish Science MPD Programme co-financed by the EU European Regional Development Fund for the financial support. Piotr Natkański acknowledges the financial support from DOCTUS scholarship cofounded by the European Social Fund. The research was carried out with the equipment purchased, thanks to the financial support of the European Regional Development Fund in the framework of the Polish Innovation Economy Operational Program (contract no. POIG.02.01.00-12-023/08).

Open Access This article is distributed under the terms of the Creative Commons Attribution Noncommercial License which permits any noncommercial use, distribution, and reproduction in any medium, provided the original author(s) and source are credited. 


\section{References}

1. Ryu Z, Rong H, Zheng J, Wang M, Zhang B. Microstructure and chemical analysis of PAN-based activated carbon fibers prepared by different activation methods. Carbon. 2002;40:1144-7.

2. Sun J, Wang Q. Effects of activation temperature on the properties and structure of PAN-based activated carbon hollow fiber. J Appl Polym Sci. 2006;100:3778-83.

3. Chiang YC, Lee CC, Lee HC. Characterization of microstructure and surface properties of heat-treated PAN-and rayon-based activated carbon fibers. J Porous Mater. 2007;14:227-37.

4. Szychowski D, Pacewska B, Makomaski G, Zieliński J, Ciesińska W, Brzozowska T. Adsorption and DSC study of mineral-carbon sorbents obtained from coal tar pitch-polymer compositions. J Therm Anal Calorim. 2011. doi:10.1007/s10973-010-1257-z.

5. Grzyb B, Albiniak A, Broniek E, Furdin G, Marêché JF, Bégin D. $\mathrm{SO}_{2}$ adsorptive properties of activated carbons prepared from polyacrylonitrile and its blends with coal-tar pitch. Micropor Mesopor Mater. 2009;118:163-8.

6. Lu CY, Wey MY. Simultaneous removal of VOC and NO by activated carbon impregnated with transition metal catalysts in combustion flue gas. Fuel Proc Technol. 2007;88:557-67.

7. Huang G, Shi JX, Langrish TAG. Removal of $\mathrm{Cr}(\mathrm{VI})$ from aqueous solution using activated carbon modified with nitric acid. Chem Eng J. 2009;152:434-9.

8. Molenda M, Dziembaj R, Piwowarska Z, Drozdek M. A new method of coating powdered supports with conductive carbon films. J Therm Anal Calorim. 2007;88:503-6.

9. Gribanov AV, Sazanov YN. Polyacrylonitrile: Carbonization problems. Russ J Appl Chem. 2008;81:919-32.

10. Kuśtrowski P, Janus R, Kochanowski A, Chmielarz L, Dudek B, Piwowarska Z, Michalik M. Adsorption properties of carbonized polyacrylonitrile deposited on $\gamma$-alumina and silica gel by precipitation polymerization. Mater Res Bull. 2010;45:787-93.

11. Van Bavel E, Cool P, Aerts K, Vansant EF. Plugged Hexagonal templated silica (PHTS): an in-depth study of the structural characteristics. J Phys Chem B. 2004;108:5263-8.

12. Janus R, Kuśtrowski P, Dudek B, Piwowarska Z, Kochanowski A, Michalik M, Cool P. Removal of methyl-ethyl ketone vapor on polyacrylonitrile-derived carbon/mesoporous silica nanocomposite adsorbents. Micropor Mesopor Mater. 2011;145:65-73.

13. Barbosa MN, Araujo AS, Galvao LPFC, Silva EFB, Santos AGD, Luz GE, Fernandes VJ. Carbon dioxide adsorption over DIPA functionalized MCM-41 and SBA-15 molecular sieves. J Therm Anal Calorim. 2011;106:779-82.

14. Xue T, McKinney M, Wilkie C. The thermal degradation of polyacrylonitrile. Polym Degrad Stab. 1997;58:193-202.

15. Jing M, Wang C, Wang Q, Bai Y, Zhu B. Chemical structure evolution and mechanism during pre-carbonization of PAN-based stabilized fiber in the temperature range of $350-600{ }^{\circ} \mathrm{C}$. Polym Degrad Stab. 2007;92:1737-42.

16. Rahaman MSA, Ismail AF, Mustafa A. A review of heat treatment on polyacrylonitrile fiber. Polym Degrad Stab. 2007;92: 1421-32.

17. Katok KV, Tertykh VA, Yanishpolskii VV, Ragulya AV, Klimenko VP, Klymchuk DO. Synthesis and investigation of barium titanate nanoparticles covered with silica shell. J Therm Anal Calorim. 2010;101:725-8.

18. Setnescu R, Jipa S, Setnescu T, Kappel W, Kobayashi S, Osawa Z. IR and X-ray characterization of the ferromagnetic phase of pyrolysed polyacrylonitrile. Carbon. 1999;37:1-6.

19. Wu CR, Salaneck WR. X-ray photoelectron spectroscopy of polyacrylonitrile. Synth Met. 1986;16:147-59.

20. Wang PH, Hong KL, Zhu QR. Surface analysis of polyacrylonitrile-based activated carbon fibers by X-ray photoelectron spectroscopy. J Appl Polym Sci. 1996;62:1987-91.

21. Pels JR, Kapteijn F, Moulijn JA, Zhu Q, Thomas KM. Evolution of nitrogen functionalities in carbonaceous materials during pyrolysis. Carbon. 1995;33:1641-53.

22. Weidenthaler C, Lu AH, Schmidt W, Schüth F. X-ray photoelectron spectroscopic studies of PAN-based ordered mesoporous carbons (OMC). Micropor Mesopor Mater. 2006;88:238-43.

23. László K, Tombácz E, Josepovits K. Effect of activation on the surface chemistry of carbons from polymer precursors. Carbon. 2001;39:1217-28. 